\section{Importance of Multifidus Muscle Activity as a Treat- ment Outcome Measure in Chronic Low Back Pain}

Sir,

Low back pain (LBP) remains the leading cause of reduced function and years lived with disability worldwide. However about $20 \%$ of global population suffers with chronic LBP (CLBP). The pain intensity measurement is a common treatment outcome measure used in the clinical practice for different musculoskeletal conditions including LPB and is relied on self-reported rating scales. ${ }^{1}$ Differences in intensity of pain do not achieve meaningful level of clinical picture of LBP cases when interventions are compared within, and between groups. Therefore, both the healthcare provider, and the patient should consider the addition treatment outcome measures to get visual feedback of their choice of LBP treatment. ${ }^{2}$ One of the Important treatment outcomes is multifidus muscle activity assessment. Introducing this as treatment outcome for patients with spinal conditions, and LBP, is important as there is evidence that the multifidus muscles are important stabilisers of lower back, and its dysfunction can strongly associate with LBP. This dysfunction could be persistent after resolving the pain leading to high recurrence, and chronicity of LBP. It is suggested that its activation is an important feature of any clinical approach to the LBP patient, and is generally targeted to be improved through rehabilitation programme. ${ }^{3}$

It is an outcome measurement that not only allows health care provider to observe multifidus muscle contraction at rest including hyperactivity, spasms, and lack of inhibition; but also provides assessment of different aberrant patterns of its activation during CLBP rehabilitation. ${ }^{3}$ A clinical perspective has also found that it is effective to use assessment of multifidus activity in management of CLBP along with other assessment. ${ }^{1}$ Moreover, a systemic review concluded to include this treatment outcome measure during rehabilitation programme of CLBP. ${ }^{4}$ There is evidence that improvement in multifidus activity can further decrease pain, and disability in CLBP. ${ }^{5}$ This assessment provides a prospect to lay the foundation for a positive alliance with treatment, which refers to a key factor in determining rehabilitation outcomes. ${ }^{1}$

Multifidus activity is being measured with several methods in Pakistan like magnetic resonance imaging, computed tomography, ultrasound, and electromyography. The ultrasound and surface electromyography are easy to use. Healthcare providers should use suitable methods which could be reached easily, and feasible for patients. It may help improve efficacy of CLBP management by demonstrating outcomes, specifically in visual way.

\section{CONFLICT OF INTEREST:}

The authors declared no conflict of interest.

\section{AUTHORS' CONTRIBUTION:}

AAMB: Conception, design of work, critical analysis and final approvalfor publishing.

BA: Conception, drafting of work, critical revision and final approvalfor publishing.

\section{REFERENCES}

1. Tagliaferri SD, Miller CT, Owen PJ, Mitchell UH, Brisby $H$, Fitzgibbon $B$, et al. Domains of chronic low back pain and assessing treatment effectiveness: A clinical perspective. Pain Practice 2020; 20(2):211-25. doi: 10.1111/papr.12846.

2. Booth J, Moseley GL, Schiltenwolf M, Cashin A, Davies M, Hübscher $M$. Exercise for chronic musculoskeletal pain: $A$ biopsychosocial approach. Musculoskeletal Care 2017; 15(4):413-21. doi: 10.1002/msc.1191.

3. Sakai $Y$, Matsui $H$, Ito $S$, Hida $T$, Ito K, Koshimizu H, et al. Electrophysiological function of the lumbar multifidus and erector spinae muscles in elderly patients with chronic low back pain. Clinical Spine Surgery 2019; 32(1):13-9. doi: 10.1097/BSD.0000000000000709.

4. Cuellar WA, Wilson A, Blizzard CL, Otahal P, Callisaya ML, Jones $\mathrm{G}$, et al. The assessment of abdominal and multifidus muscles and their role in physical function in older adults: $A$ systematic review. Physiotherapy 2017; 103(1):21-39. doi: 10.1016/j.physio.2016.06.001.

5. Rezazadeh F, Taheri N, Okhravi SM, Hosseini SM. The relationship between cross-sectional area of multifidus muscle and disability index in patients with chronic nonspecific low back pain. Musculoskeletal Sci Prac 2019; 42:1-5. doi: 10.1016/j.msksp.2019.03.005.

Aftab Ahmed Mirza Baig ${ }^{1}$ and Basit Ansari

${ }^{1}$ Department of Physiotherapy, Dow University of Health Sciences, Karachi, Pakistan

${ }^{2}$ Department of Health, Physical Education, and Sports Sciences, University of Karachi, Karachi, Pakistan

Correspondence to: Dr. Aftab Ahmed Mirza Baig, Department of Physiotherapy, Dow University of Health Sciences, Karachi, Pakistan

E-mail: ab.dptrm@gmail.com

Received: November 05, 2020; Revised: December 05, 2020; Accepted: December 28, 2020

DOI: https://doi.org/10.29271/jcpsp.2021.12.1528 